\title{
Research Progress on the Wing and Veins of Diptera Phoridae
}

\author{
Bingbing Cui \\ Shenyang University, Shenyang 110044, China \\ Tel: 86-24-2324-3799Ｅ-mail: bingb-ndb@163.com
}

\begin{abstract}
Wing is one of the most important organs of insects, and due to wings, insects adapt to different environmental conditions in the world. Consequently, wings played a critical role in the evolution of insects. Different types of wings reflected different evolutionary patterns and rate.
\end{abstract}

Keywords: Eye fly, Wing, Veins

\section{Introduction}

Phoridae belongs to Phoridae superfamily, Aschiza section, Muscomprpha suborder and Diperta order, and one of the biggest suborders of Diperta. Approximately 3,400 species are known in 250 genera. They are a diverse and successful group of insects, with very obvious species differentiation. They are of great application values and worthwhile to get further investigation. Their own specific morphological characteristics suggested that "Phoridae definitely doesn't belong to Muscomphrpha, and can't find their relatives in straight split class" (Liu, 2001, PP. 6-8). As seen from the perspective of species evolution, eye flies belong to the specie group between mosquito or horsefly and flies, and intensive and extensive researches on eye flies means to profound and further investigations on the evolutionary relationship of total Diptera and are of important guiding significance for the studies of specie evolution (Disney, 1990a).

\section{Overview of eye fly wing}

Both genders in the vast majority categories of Phoridae have well-developed wings, and females of several few categories have short-fin wings, wing buds or no wings. Wings usually are membranaceous transparent, ranging from colorless to brown, and their surface mostly doesn't possess scale and gross. Veins facies in Muscomprpha is particularly characteristic, and of great significance in taxonomy. Wing is one of the most important organs of insects, and due to wings, insects adapt to different environmental conditions in the world. Consequently, wings played a critical role in the evolution of insects. Different types of wings reflected different evolutionary patterns and rate (Zheng, 1987, PP. 130-164).

\section{Research significance on wings and veins}

Both genders in the vast majority categories of Phoridae have well-developed wings, and females of several few categories have short-fin wings, wing buds or no wings. Wings usually are membranaceous transparent, ranging from colorless to brown, and their surface mostly doesn't possess scale and gross. Veins facies in Muscomprpha is particularly characteristic, and of great significance in taxonomy. Wing is one of the most important organs of insects, and due to wings, insects adapt to different environmental conditions in the world (Liu, 2005, PP. 314-317). Consequently, wings played a critical role in the evolution of insects. Different types of wings reflected different evolutionary patterns and rate.

\section{Current research status of wings and veins}

Insect wings was composed of membranes, veins and several cross veins, and formed a kind of solid structure similar to truss structures. Among them, veins longitudinally extended outside along the wings and sustained the membranes, and cross veins connected all longitudinal veins with one another together (Cai, 2001). Among various types of insects, they all have their own unique distribution patterns of veins, namely venation. In China, after morphological comparison posterior wings in Coleoptera are named and morphological comparison is undertaken according to the posterior vein distribution situation and its folding patterns. The denomination of posterior wing veins mainly referred to the previous researches by scholars such as Comstock (1898), Needham (1899), Snodgrass (1935), Kukalova2Peck (1993), Lawrence (2004) and so on, meanwhile, in addition to the similar researches on the groups of Coleoptera initiated by Chinese scholars in recent years (Wang, 2000; Ge et al, 2003; Liu et al, 2005).Taking posterior wings of Mylabris calida Pallas as example, using the naming system of Kukalova2Peck and Lawrence (2004), according to the relationship between primary vein and wing fold, the 
wing was divided into six zones, and the features of veins were described. Yang et al had selected 11 representative species from Mylarbris, and investigated their morphological features of posterior wings through comparison; Mylabris calida Palla, 1782 as a model, the posterior wing veins of Mylabris were discussed and their characters were described (Yang, 2007, PP. 429-434).

Sun Yuan and Wang Mingfu gave a basic elaboration of insect wings from six aspects, viz. origin, basic structure, pulse phase, type, chain and function. Moreover, Ge Silin, Yang Xingke et al launched a comparative morphological study on posterior wings of 20 species from 20 genera in chrysomelinae, mainly discussed the names and changing types of veins, depicted their model map, morphological characteristics figure of 20 kinds of veins, and veins changing type figure. Their evolutionary significance was primarily concluded, and the most original posterior wing type in chrysomelinae was considered as follows: large aspect ratio, cross veins cv existed between $\mathrm{Cu}_{\mathrm{la}}$ and $\mathrm{Cu}_{\mathrm{lb}}$ or cp between $\mathrm{Cu}_{\mathrm{lb}}$ and $\mathrm{Pcu}, \mathrm{Cu}_{\mathrm{lb}}$ was partly separated from Pcu end, and failed to form an integrated end. In the present paper, based on the previous researches, we attempted to launch a further investigation on the veins and their phase in chrysomelinae by using the naming system of Suzuki (1994). Liu Shanshan and Liu Guodong had launched a comparative morphological study on the posterior wings of insects in Uloma, and observed and analyzed the posterior wings of 16 kinds of insects in Uloma, using Uloma excise Gebien as a model. They preliminarily discussed their names, gave a brief summary of their characteristics, and also depicted the morphological characteristic diagram of 16 kinds of posterior wing veins in Uloma.

Li Youlian and Cao Hui had investigated the changing rules of chaitophorus populialbae veins, the results indicated that environmental conditions (mainly temperature) played a cardinal role in the changing of veins; due to the suitable weather in May, a large number of chaitophorus populialbae propagated with a greater population density, and vein changing tended to be moderate. From the last ten days of May to early June, temperature increased suddenly with severe changes, population density declined, and due to the unfavorable environmental conditions, vein differentiation tended to be dramatic; After the second ten days of June, with the constant and stable high temperature and plenty of rainwater, aphids mostly appeared in the lower leaves near to the moist ground with great population density, and due to the appropriate environmental conditions, vein differentiation tended to be modest. Chaitophorus populialbae vein differentiation had a general trend, namely from less to more, variation cardinally occurred in the midveins, and through displacement merge or disappearance, veins varied towards of binary-type midveins with a trend of single midvein development. At the same time, regression signs of $\mathrm{Cu}_{1}, \mathrm{Cu}_{2}, \mathrm{R}$ veins were also observed. The phenomenon that veins evolved from less to more was compatible with their biological characteristics. Aphids with wings rarely moved, generally crawled and spread nearby and seldom flew.

According to the law of use and disuse in Charles Darwin's theory of evolution ( $\mathrm{Li}, 1963$ ), chaitophorus populialbae wings belonged to the degrading organs, and not a major kinetic organ, and veins thus decreased gradually. It was a quite important discovery, and of certain significance in ecology, genetics and taxonomy. Wang Wenkai had studied the posterior wing veins in Lamiinae, and through the comparison of 152 species in 18 genera of Lamiinae, and found that the characteristics of posterior wing veins had significant differences among different species or genus with tribal and genus significance in taxonomy and critical values in elaborating the genetic relationship and systematic evolution in different species of Lamiinae.

Zhao Yanru and Tong Jin had launched a micro-structure investigation on housefly forewing vein and the results showed as follows: simple venation distributed in housefly membranous wings; according to the analysis of angels among veins from a mechanical perspective, microtrichia closely distributed in both sides of membranous wings; according to the structure analysis of cross-section, housefly membranous wings were winkle with hollow veins. Studies on the insect membranous wings would provide technological references to biomimetic design of new two-dimensional composite materials. Pan Pengliang, Shen Zuorui et al had launched extraction experiments of insect wing configuration and vein characteristics using DrawWing software with its two different versions, viz. DOS and Windows. Xu Jinjiang, Luo Xinfu, and Tang Linhua had compared the wing spot conformation between anopheles minimus and anopheles fluviatilis, and whether male or female, there was obvious significance in wing spot conformation between anopheles minimus and anopheles fluviatilis. It could be speculated that wing spot conformation was the important basis to identify anopheles minimus and anopheles fluviatilis.

Tan Ken had investigated effects of western honeybee larval growth temperature on adult wing morphology, and put Apis mellifera near to the pupal period into incubators at a constant temperature condition of $32^{\circ} \mathrm{C}, 35^{\circ} \mathrm{C}$ and $36^{\circ} \mathrm{C}$, respectively till emergence. Standard morphological characteristics of honeybee wings were measured, including size, cubitus length and 11 wing cubitus angles, and all resultant data carried through comparative analysis of multiple variables, correlation analysis, principal component analysis and discriminant analysis. The 
results showed that different temperature conditions had a significant impact on the morphological characteristics of honeybee wings.

Zhou Changfa had studied the existing mayfly wing base longitudinal veins trends and healing pattern, and found that the trends and healing situation varied strikingly in most species, and its original condition was tough to speculate and could be only traced only in very few species that still remained several visible vestige. Chinese siphluriscus chinensis wing base remained trend traces of independent subcosta brace, several midvein $\mathrm{M}$ and cubitus $\mathrm{Cu}$ backbone. Based on these, in combination with the characteristics related to wing base of Ephemera rufomaculata and Ephemera rufomaculata, he brought forward the major longitudinal vein base trend and basic healing pattern in mayflies and its main points were as follows: midvein and radial vein backbone exhaled at the base, first approached to each other or healed up and then separated, they both divided into two posterior premedia and radial sector respectively, they also first healed up and then separated, and the cubituses were independent all the way. Such pattern that midvein and radial vein first healed up and then separated was extremely similar to the situation of neoptera, but quite different from the dragonfly (midvein and radial vein healed up at the base in dragonflies).

The role played by subcosta brace was believed to strengthen the links among longitudinal veins that separated from one another due to the well-developed wing base bone plates. Through the aphid wing dimorphism studies, Wang Kai, and Wang Haiyan et al elaborated the biological characteristics of aphids and phylogenetic distribution and impact factors of its wing dimorphism, and analyzed the evolution traits of its wing dimorphism. Based on comparative morphological studies, Wang Xinli, Bao Rong and Wan Xia had demonstrated the homology of front or back venation backbone in antlions, and put forward a new naming system of its venations. Homology demonstration based on five aspects as follows: 1) comparative analysis between existing antlion venations and its fossil data; 2) comparative analysis between antlion venation and its hypothetical model; 3) comparative analysis between antlions and other insect venation; 4) comparative analysis between front and back wing characteristics in antlions; 5) comparative analysis of the existing name system in antlion venations. This new naming system could alleviate the complexity of morphological description of antlion and the difficulty to understand the characteristics tremendously.

Currently, few researches on the wings of eye flies have been documented in the world. We analyzed the morphological characteristics of wings in the light of previous studies, and depicted their properties. We also discussed their genetic relationship, primarily elaborated the significance of their wings in evolution, and filled the research gap in this field.

\section{References}

Cai, W.Z. (2001). General entomology. Beijing: China Agricultural University Press.

Liu, G.C. (2001). Chinese eye fly classification. Shenyang: Northeastern University Press, 6-8.

Liu, G.C., Fang, H., \& Zhu, W., et al. (2005). Preliminary analysis of insect fauna in China Phoridae. Classification and Diversity of Insects in China, 50 (4):314-317.

Yang, Y.X., \& Ren, G.D. (2007). Hind-wing morphology of Mylabris Fabricius (Coleoptera:Meloidae) from China. Acta Entomologica Sinica, 50 (4):429-434.

Zheng, L.Y. (1987). Animal classification principles and methods. Beijing: Higher Education Press, 130-164. 